\title{
Study on the Course and Speed Cooperative Control Method for Unmanned Surface Vehicle
}

\author{
S.J. CAO, Y.T.CHEN and F.M.ZENG* \\ College of Power Engineering, Naval University of Engineering, Wuhan, China \\ 975526435@qq.com
}

\begin{abstract}
Keywords: unmanned surface vehicle; fuzzy algorithm; self-adaptive control; cooperative optimization.

Abstract. In order to solve the problem of autonomous dynamic control for unmanned surface vehicle (USV) under the unknown environment, the course and speed cooperative control method based on adaptive fuzzy algorithm is studied in this paper. A fuzzy control algorithm is designed of which the inputs are the deviation of course and position and the outputs are the rudder angle and throttle. The self-adaptive control method is used, of which the input is the deviation rate of course and the output is the control interval, to make system sensitive with external changes. The objective function of the optimization is the weighted minimum of the arrival time and the change frequency of rudder. The control effect of the parameters like domains has been analyzed. The results offer some suggestions for the optimization of parameters of the control system.
\end{abstract}

\section{Introduction}

The Unmanned Surface Vehicle (hereinafter referred to as USV) is an unmanned surface ship, which belongs to the field of unmanned intelligent power platform research. The autonomous motion control is one of the core technologies that USV distinguishes one of the manned ship.

Traditional USV control methods are: (1) Control algorithm. Based on accurate mathematical model of the conventional control algorithm, ship motion model is difficult to precisely determined, and in the water environment of uncertainty and strongly nonlinear and linear time invariant motion model parameters to changes in real-time and accurate description of the ship motion state. (2) Control strategy. Course and speed of the cooperative control level is weak, the main consideration with course of motion control result, only will be heading into the scope of autonomous control algorithm, and less consideration of the impacts of speed of motion control rapidity, robustness and autonomy.

This paper will address the inadequacy of existing technologies. A cooperative control method based on fuzzy adaptive algorithm of USV course and speed is studied, which avoid conventional methods of ship motion modeling problem, and improve fuzzy control strategy and algorithm, and incorporate adaptive factors, finally improve the control method under the uncertain water environment adaptability and robustness.

\section{Course and speed of the cooperative control method}

Coope rative control model. Fig. 1 is the model of the cooperative control of the USV course speed, and the design frame of the test platform software. The specific control method is divided into two sub threads, corresponding to fuzzy control and adaptive control method.

In the fuzzy control thread, the fuzzy control algorithm for the solution of heading angle deviation $\mathrm{P}$ and straight-line distance deviation $\mathrm{D}$ is calculated by the fuzzy set membership and output rudder angle deflection control variable $\mathrm{U}$ and throttle opening control volume $\mathrm{V}$.

In the adaptive control thread, the heading angle deviation rate, which means $\mathrm{Pt}=\mathrm{P} / \mathrm{t}$, is computed and divided into three categories. The control period for high heading deviation is shorter. Conversely, a longer cycle of control is used to achieve self-suitable for adjusting the parameters of fuzzy controller which is in response to changes in the external environment. 


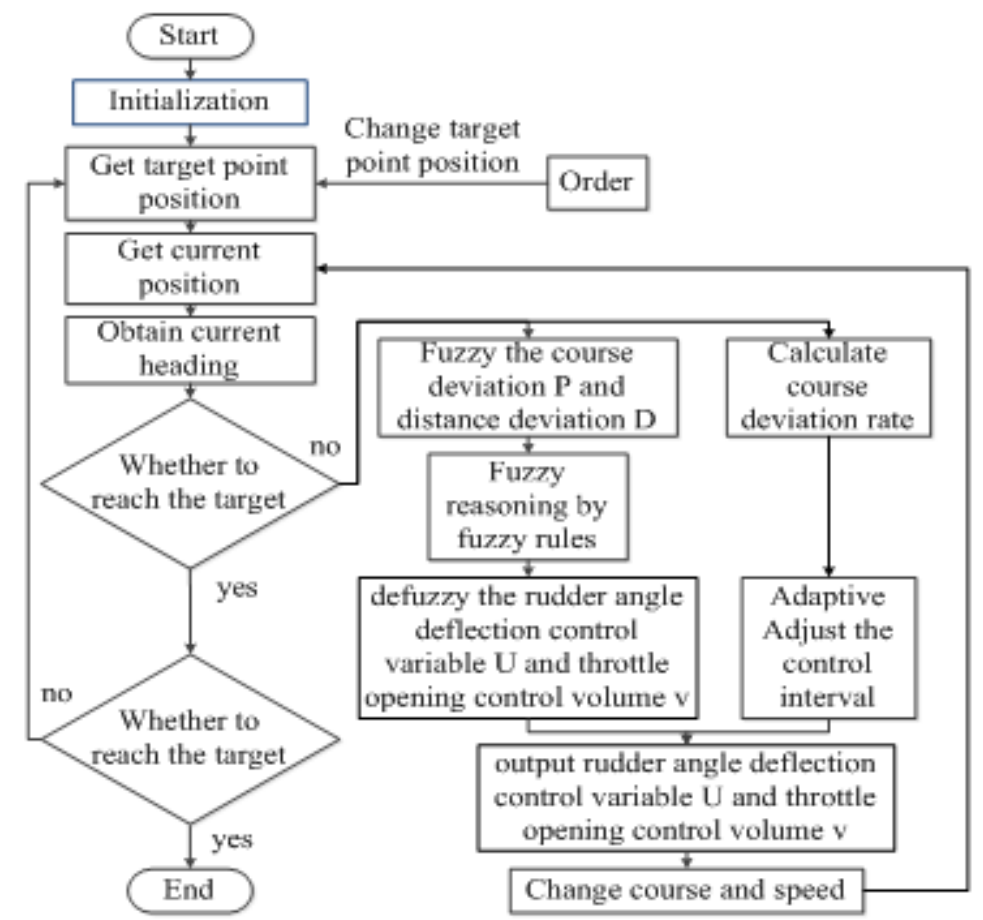

Figure 1. The model of the course and speed cooperative control method.

Fuzzy adaptive algorithm. The fuzzy adaptive algorithm is based on simulation experiment and expert experience to develop the function parameters and fuzzy rules, and adaptive adjust itself with the acquisition of the change of the external environment.

First, the input of the algorithm is obtained by the data pre-processing. Acquired by the sensor position and angle information and the initial settings can get the longitude direction distance deviation $X$ (set the east as the positive direction) and latitud inaldistance deviation of $Y$ (se the north as the positive direction), heading angle $\theta$ (set the north as the direction for reference). The heading angle de viation $\mathrm{P}$ and straight- line distance de viation $\mathrm{D}$ can be calculated from the following formula:

$$
\begin{aligned}
& \mathrm{P}= \begin{cases}\theta+\tan ^{-1}\left(\frac{Y}{X}\right)+90 & X \leq 0 \\
\theta+\tan ^{-1}\left(\frac{Y}{X}\right)-90 & X>0\end{cases} \\
& \mathrm{D}=\sqrt{\left(X^{2}+Y^{2}\right)}
\end{aligned}
$$

Second, the heading angle deviation $\mathrm{P}$ and straight-line distance deviation $\mathrm{D}$ respectively are introduced into their membership function fuzzy processing. At the same time, the output rudder angle deflection control variable $\mathrm{U}$ and throttle opening control volume $\mathrm{V}$ are also respected by the corresponding fuzzy set and membership function characterization. Define the type:

$$
A_{j}=\left\{x, \mu_{A_{j}}(x) \mid x \in R\right\}
$$

as a fuzzy set, where A represents the collection of fuzzy quantities, the following is represented by D, $\mathrm{U}, \mathrm{P}, \mathrm{V}$. The different values of the identification number $\mathrm{J}$ correspond to different fuzzy partitions. The independent variable of the fuzzy set is $\mathrm{x}$, and $\mathrm{R}$ means the domain of $\mathrm{x}$, which is for its value range. In this paper, for the course deviation angle and rudder angle deflection, $\mathrm{j}=-2,-1,0,1,2$ are respectively on behalf of NB (negative big deviation/left rudder deflection), NS (negative small deviation/left rudder deflection), ZE (zero bias/zero rudder angle), PS (positive small deviation/right rudder deflection), PB (positive big deviation/right rudder deflection). Similarly, for the straight line distance deviation $\mathrm{D}$ and throttle opening control volume $\mathrm{V}, \mathrm{j}=0,1,2$ are respectively on behalf of the $\mathrm{Z}$ (zero bias), S (small deviation), B (big deviation). 
After the input quantity $\mathrm{D}$ and $\mathrm{P}$ are pasting, the output variables $\mathrm{V}$ and $\mathrm{U}$ are inferred from the corresponding fuzzy rules, and the Tab. 1 and Tab. 2 are the rule tables for the reasoning process:

Table 1. The fuzzy reasoning rule of deflection control of rudder angle

\begin{tabular}{c|c|c|c|c|c}
\hline $\mathrm{U}$ & $\mathrm{NB}$ & $\mathrm{NS}$ & $\mathrm{ZE}$ & $\mathrm{PS}$ & $\mathrm{PB}$ \\
\hline $\mathrm{B}$ & $\mathrm{PS}$ & $\mathrm{PB}$ & $\mathrm{ZE}$ & $\mathrm{NB}$ & $\mathrm{NS}$ \\
$\mathrm{S}$ & $\mathrm{PB}$ & $\mathrm{PS}$ & $\mathrm{ZE}$ & $\mathrm{NS}$ & $\mathrm{NB}$ \\
$\mathrm{Z}$ & $\mathrm{ZE}$ & $\mathrm{ZE}$ & $\mathrm{ZE}$ & $\mathrm{ZE}$ & $\mathrm{ZE}$ \\
\hline
\end{tabular}

Table 2. The fuzzy reasoning rule of opening control of throttle

\begin{tabular}{c|c|c|c|c|c}
\hline V & NB & NS & ZE & PS & PB \\
\hline B & S & B & B & B & S \\
S & S & S & B & S & S \\
Z & Z & Z & Z & Z & Z \\
\hline
\end{tabular}

Fuzzy reasoning process adopts Mamdani rules. For example, the output $\mathrm{z} \in \mathrm{U}$ was obtained by the input $\mathrm{x} \in \mathrm{P} 、 \mathrm{y} \in \mathrm{D}$ through the reasoning process, two fuzzy control rules are as follow:

$$
\begin{aligned}
& R 1 \text { : If } x \text { is } P 1 \text { and } y \text { is D1 then } z \text { is } U 1 \\
& R 2 \text { : If } x \text { is } P 2 \text { and } y \text { is D2 then } z \text { is } U 2
\end{aligned}
$$

Inference operation is shown in the following formula:

$$
\begin{aligned}
& \omega_{i}=\max _{x} \mu_{P_{i}}(x) \wedge \max _{y} \mu_{D_{i}}(y) \\
& \mu_{U}(z)=\left[\omega_{1} \wedge \mu_{U_{1}}(z)\right] \cup\left[\omega_{2} \wedge \mu_{U_{n}}(z)\right]
\end{aligned}
$$

where the symbol " $\wedge$ " refers to the Cartesian product, namely the intersection of small operations. The intermediate variable is the minimum result of the maximum of the membership degree of $Y$ and $\mathrm{X}$ in the respective fuzzy sets. The union of function curve of output membership of the $\omega$ interception. At the end of the output curve is introduced into defuzzification, namely the centroid coordinate of area:

$$
z_{0}=\frac{\int z \mu_{U}(z) d z}{\int \mu_{U}(z) d z}
$$

Before entering the next control loop, the adaptive method adjusts its parameters in order to improve the robustness of the algorithm and speed up the convergence of the control effect. In this paper, the adaptive adjustment of the control period is carried out. The heading deviation rate is $\mathrm{Pt}=\mathrm{P} / \mathrm{t}$, which is divided into three levels by its value, to represent the severe degree of the control environment. When the rate is high, we regard it as a complex control environment and employ the highest control interval; when the rate is low, we regard it as a peace control environment and employ the lowest control interval; the rest is as the conventional control environment and the economic control interval is put to use. In this way, the control effect can be improved with strong anti-interference ability in the complicated sea conditions, also be improved with the calculation precision and stability and prolong the using time in quiet environment.

\section{Optimization of the control parameters}

In the adaptive fuzzy control algorithm, control parameters include input and output's domain, control interval, fuzzy inference rules, etc., in which the input's domain is key parameter to control effects. 
In the process of completing the task of USV, the arrival time of the target point and the change frequency of rudder are two important tasks. The weighted minimum of them can be the objective function, as shown in the following formula:

$$
f\left(x_{1}, x_{2}, x_{2} \cdots x_{n}\right)=\alpha t\left(x_{1}, x_{2}, x_{a} \cdots x_{n}\right)+\beta s\left(x_{1}, x_{2}, x_{a} \cdots x_{n}\right)
$$

where $t(x)$ is the function of the arrival time, and $s(x)$ is the function of the change frequency of rudder, and $\mathrm{x}$ is the controller parameters, and $\alpha, \beta$ is two parameters' weight. The parameter optimization take the minimum of the objective function as the target to improve the efficiency of navigation and reduce the mechanical strain.

Fig. 2 is the track of USV when the input's domain changes. The target position is 50 meters away from USV initial position, and USV initial heading angle is $90^{\circ}$. In the figure, the dash line circle means arrival circle and has a radius of 5 meters.

In Fig. 3, 4, there are the relationships between the input's domain and the optimization object. Fit the simulation results and get the analytic expression of $\mathrm{t}(\mathrm{x})$ and $\mathrm{s}(\mathrm{x})$. We can see that arrival time $\mathrm{t}$ and the input's domain are positively correlated while the change frequency of rudder $\mathrm{s}$ and the input's domain are negative correlated from the figures. Take two analytic expressions into equation (7), and the $\mathrm{x}$ value will be the best input's domain solution when $\mathrm{f}(\mathrm{x})$ takes the minimum value.

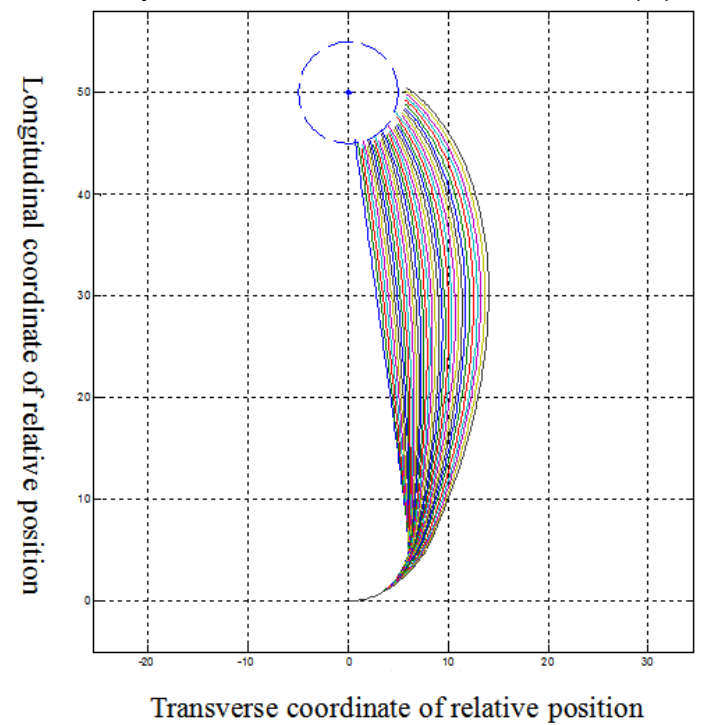

Figure 2. The track figure of USV when the input's domain changes

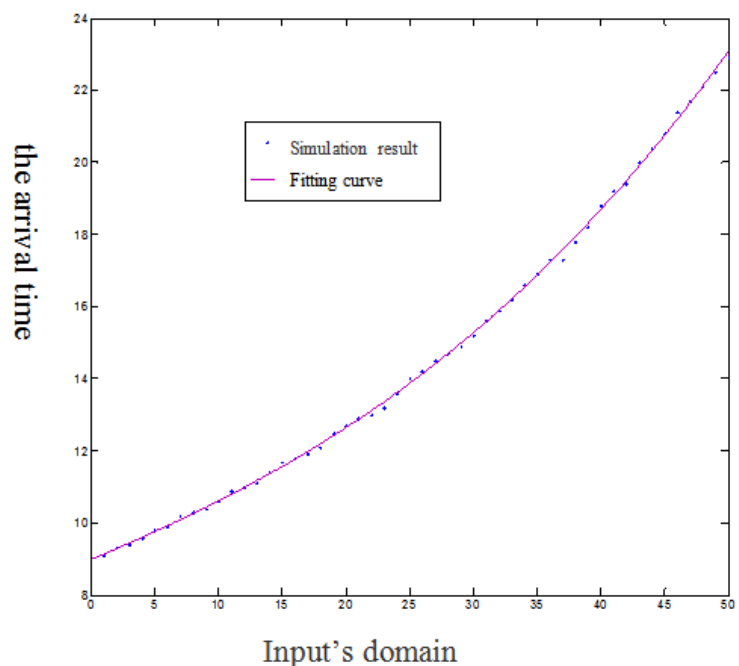

Figure 3. The relationship between the arrival time and the input's domain 


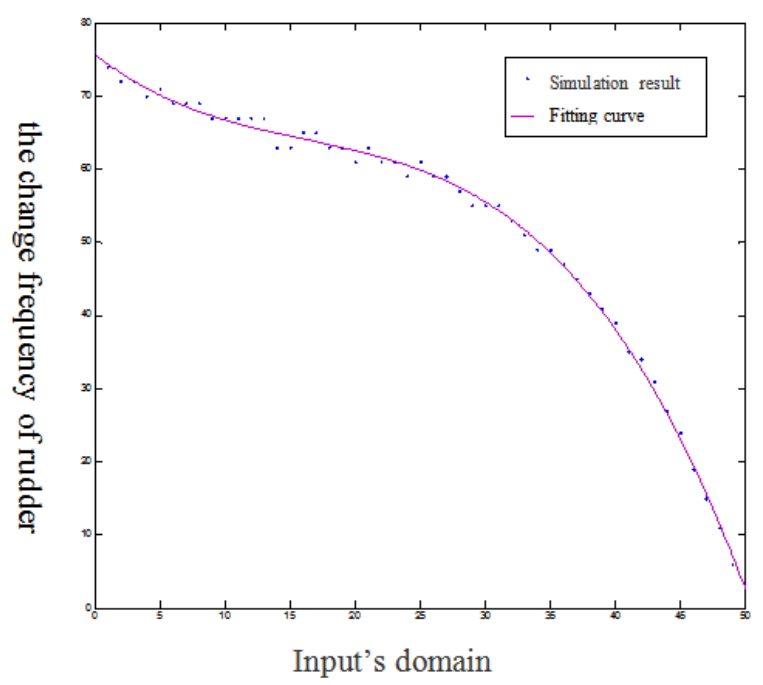

Figure 4. The relationship between the change frequency of rudder and the input's domain.

The results of the optimal analysis of control parameters show that: (1) The performance parameters of control model is the arrival time and the change frequency of rudder and the optimization parameters are input's domain. The arrival time and the input's domain are positively correlated while the change frequency of rudder and the input's domain are negative correlated; (2) When the relationship curve has a big fluctuation and curve fitting's accuracy is not high, the weight of function should be appropriate to reduce; (3) When there is only a single optimization variable, objective function $\mathrm{f}(\mathrm{x})$ is bounded and convergence and can obtain the minimum value, indicating that the optimization method is universal for all the control parameters. Thus, the optimization method can effectively find the suitable controller parameters.

\section{Conclusion}

In this paper, the course and speed cooperative control method is studied. The advantages of fuzzy control algorithm are used to control both the course and speed of the USV with adding adaptive control thread to response to external environment by itself. Through the simulation and optimization analysis of the control system of the platform, a set of effective optimization scheme is obtained, which provides a theoretical basis for the further experimental research.

\section{References}

[1] YAN Rujian, PANG Shuo, SUN Hanbing, PANG Yongjie. Development and missions of unmanned surface vehicle [J].Journal of Marine Science and Application, 2010, 9(4):451-457.

[2] DO K D,PAN Global robust adaptive path following of underactuated ships [J].Automatica, 2006, 42(10):1713-1722.

[3] CHEN M. The hydrodynamic models and the decoupling control research in the speed \& course of USV [D].Harbin: Harbin Engineering University, 2011:1-23.

[4] CHEN S L, CHEN H. Modeling and simulation based on fuzzy neural network for unmanned surface vehicle[J].Ship Science and Technology.2010, 32(11): 134-136.

[5] Caccia M, Bono R, Bruzzone G. Design and exploitation of an autonomous surface vessel for the study of sea-air interactions[C]. Proceedings of the 2005 IEEE International Conference on Robotics and Automation, Barcelona, Spain, 2005:3582-3587.

[6] Caccia M, Bono R, Bruzzone G, et al. An autonomous craft for the study of sea-air interactions [J].IEEE Robotics \& Automation Magazine.2005, 12(3):95-105.

[7] Y.Liao, L.Wan, J.Zhuang. Back stepping dynamical sliding mode control method for the path following of the underactuated surface vessel[C]. International Conference on Advanced in Control Engineering and Information Science. Amsterdam: Elsevier Science BV, 2011:256-263. 